\title{
Trouver la voie - répondre aux besoins des adultes atteints de maladies d'origine infantile
}

U n des grands succès de la médecine au cours des 25 dernières années fut de réussir à contrôler de nombreuses maladies infantiles graves qui tuaient des enfants voilà à peine une génération. Peu d'adultes étaient atteints de fibrose kystique au cours des années I980 - or, la durée médiane de survie à la fibrose kystique dépasse maintenant de loin les 30 ans au Canada. La leucémie infantile n'est plus une condamnation - plus de $80 \%$ des enfants atteints de leucémie lymphoblastique aiguë passent en rémission de longue durée, et même chez ceux qui ont une leucémie aiguë myéloblastique, le taux de survie dépasse maintenant les $50 \%$. La plupart des bébés qui naissent à 26 ou 28 semaines de gestation survivent maintenant. Chez beaucoup de nouveau-nés atteints de graves malformations congénitales, on a posé le diagnostic in utero et établi des plans de soin avant l'accouchement. Il est souvent possible de gérer, de modifier ou de corriger les malformations cardiaques, les problèmes gastro-intestinaux ou les anomalies rénales graves.

À mesure que ces survivants de maladies infantiles graves arrivent à l'âge adulte, on constate l'apparition de nouveaux problèmes parfois reliés à la maladie (diabète chez les patients adultes atteints de fibrose kystique) ou à des séquelles du traitement de la maladie (deuxième tumeur maligne chez les survivants d'un cancer infantile). Face à ces besoins, les cliniques pour adultes atteints de maladies cardiaques congénitales ou de fibrose kystique se multiplient, tout comme les cliniques de suivi des adultes qui ont survécu à un cancer infantile. Il convient de signaler que beaucoup de ces cliniques ont fait leur apparition parce que ces patients adultes ont exigé que leurs soins passent du centre de pédiatrie au milieu adulte et leur soient prodigués par des spécialistes de la maladie. Ces adultes avertis savent trop bien que des soins spécialisés multidisciplinaires particuliers à une maladie font une grosse différence sur les plans de la survie et de la qualité de vie. Même si le virage vers les milieux de soins aux adultes ne s'est pas toujours fait en douceur, la plupart des jeunes atteints de fibrose kystique, d'une maladie cardiaque congénitale ou d'un cancer s'attendent à vieillir et à recevoir leurs soins dans le cadre d'un programme pour adultes.

Les adultes atteints de graves problèmes de santé découlant de l'enfance ne sont pas tous aussi chanceux. Non seulement il existe des différences régionales d'accès aux programmes de soins aux adultes conçus pour répondre aux besoins de survivants de maladies infantiles, mais on manque aussi de programmes particuliers à certains problèmes. Par exemple, les adultes atteints de paralysie cérébrale et qui ont de graves incapacités du développement demeurent souvent marginalisés dans J le système de soins aux adultes. Des problèmes interdépendants comme une mauvaise nutrition, une dislocation de la hanche, la douleur chronique, les spasmes musculaires et les contractions, les pneumonies à répétition, la violence physique et les problèmes de communication sont abordés à la pièce et on se concentre sur un seul problème au lieu de procéder de façon coordonnée et multidisciplinaire. Dans certains cas, des problèmes comme la violence ne sont pas identifiés du tout ou on n'en tient tout simplement pas compte. Même si les cliniques multidisciplinaires pédiatriques ont prolongé la survie et amélioré la qualité de vie de ces enfants et de ces adolescents, la "progression» vers un programme de soins de santé spécialisés, multidisciplinaires et intégrés pour adultes est rarement possible, ces cliniques étant elles-mêmes très rares. Ces programmes proposés de soins tertiaires multidisciplinaires pour adultes constitueraient une ressource pour les médecins de famille qui dispensent les soins primaires continus à cette population. Le problème est devenu plus urgent parce que de plus en plus de ces enfants et adolescents atteignent l'âge adulte.

Le sort des adultes atteints de paralysie cérébrale et qui ont un retard sévère du développement met en évidence la difficulté suivante : d'une part, nous devons fournir à tous et toutes les soins de santé nécessaires pour traiter les problèmes courants de l'âge adulte et du vieillissement, mais de l'autre, nous avons besoin de soins multidisciplinaires très spécialisés pour des souspopulations d'adultes qui ont, sur le plan de la santé, des antécédents complexes et pour qui les "soins pour adultes normaux» ne suffisent pas. Comment établir un équilibre entre ces besoins divergents, particulièrement lorsqu'un groupe, soit celui des adultes atteints de paralysie cérébrale et d'un retard du développement, ne peut intervenir pour se défendre?

Comme les ressources humaines et matérielles sont limitées, le rationnement et l'établissement de priorités ont souvent mis l'accent sur les besoins des masses ou sur ceux des groupes qui se font entendre bien fort, par exemple les adultes en attente d'arthroplastie.

Comment distribuer les ressources de façon plus équitable et humanitaire? Qui devrait prendre ces décisions, et en fonction de quels critères? Ces problèmes demandent une nouvelle réflexion, un processus plus transparent où l'on tient compte de la pertinence, de l'efficacité des coûts et de l'incidence sur la qualité et la durée de la vie. Il faut clarifier les données sur lesquelles s'appuient les choix.

Le système de santé du Canada repose sur le concept du bien collectif en vertu duquel on s'efforce de dispenser des soins de qualité dans des délais raisonnables pour tous de façon à ce que personne ne soit oublié, abandonné ni poussé à la faillite à cause des coûts élevés des soins de santé nécessaires. Le rationnement des soins de santé et les décisions sur les priorités ne doivent pas oublier les besoins des marginalisés de la société, et en particulier de ceux et celles qui n'ont pas de porte-parole tels que les adultes atteints de paralysie cérébrale et de retard du développement.

\section{Noni MacDonald}

Rédactrice en chef intérim

$J A M C$

Ottawa (Ont.)

Cet article a fait l'objet d'un examen par les pairs. 